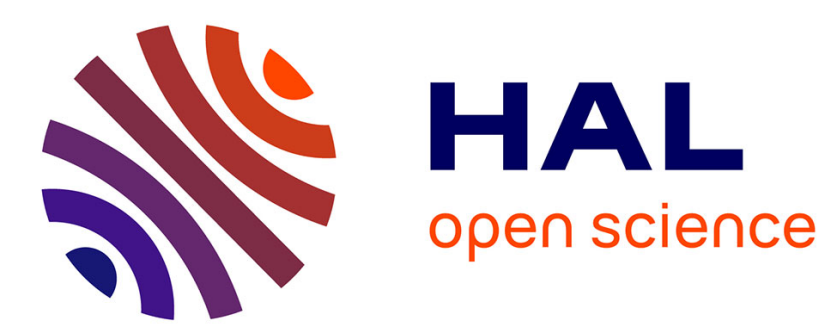

\title{
A Monte Carlo method without grid for a fractured porous domain model
}

Fabien Campillo, Antoine Lejay

\section{To cite this version:}

Fabien Campillo, Antoine Lejay. A Monte Carlo method without grid for a fractured porous domain model. Monte Carlo Methods and Applications, 2002, 8 (2), pp.129-147. inria-00152412

\section{HAL Id: inria-00152412 \\ https://hal.inria.fr/inria-00152412}

Submitted on 25 Feb 2009

HAL is a multi-disciplinary open access archive for the deposit and dissemination of scientific research documents, whether they are published or not. The documents may come from teaching and research institutions in France or abroad, or from public or private research centers.
L'archive ouverte pluridisciplinaire HAL, est destinée au dépôt et à la diffusion de documents scientifiques de niveau recherche, publiés ou non, émanant des établissements d'enseignement et de recherche français ou étrangers, des laboratoires publics ou privés. 


\section{A Monte-Carlo Method without Grid to Compute the Exchange Coefficient in the Double Porosity Model}

Fabien Campillo ${ }^{1}$ — Projet SYSDYS (INRIA/LATP)

Antoine Lejay ${ }^{1}$ — Projet SYSDYS (INRIA/LATP)

Abstract:The double porosity model allows to compute the pressure at a macroscopic scale in a fractured porous media, but requires the computation of some exchange coefficient characterizing the passage of the fluid from and to the porous media (the matrix) and the fractures. This coefficient may be numerically computed by some Monte-Carlo method, by evaluating the average time a Brownian particle spend in the matrix and the fissures. Although we simulate some stochastic process, the approach presented here does not use approximation by random walks, and then does not require any discretization.

Keywords: Monte-Carlo method, simulation of Brownian motion exit time, double porosity model, fractured porous media

AMS Classification: $76 \mathrm{~S} 05$ (65C05 76M35)

Published in Monte Carlo Methods Appl.. 8:2, 129-147, 2002

Archives, links \& reviews:

○ HAL: inria-00152412

○ MR number: MR1916913

${ }^{1}$ Current address: Projet SYSDYS (INRIA/LATP)

IMT

38 rue F. Joliot-Curie

13451 Marseille Cedex 20 (France)

E-mail: Fabien.Campillo@sophia.inria.fr Antoine.Lejay@sophia.inria.fr 


\section{Introduction}

This paper presents an algorithm of simulation of a diffusion in a fissured porous medium (the matrix). In fact, the algorithm just gives the times and position of the particle hitting the interface between the matrix and the fissures.

Let $\Omega$ be a bounded, closed subset $\mathbb{R}^{2}$, and $\Omega=\Omega_{\mathrm{f}} \cup \Omega_{\mathrm{m}}$ with $\Omega_{\mathrm{f}} \cap \Omega_{\mathrm{m}}=\emptyset$. The subset $\Omega_{\mathrm{m}}$ is the matrix, that is a porous media. The subset $\Omega_{\mathrm{f}}$ is the net of "thin" fissures.
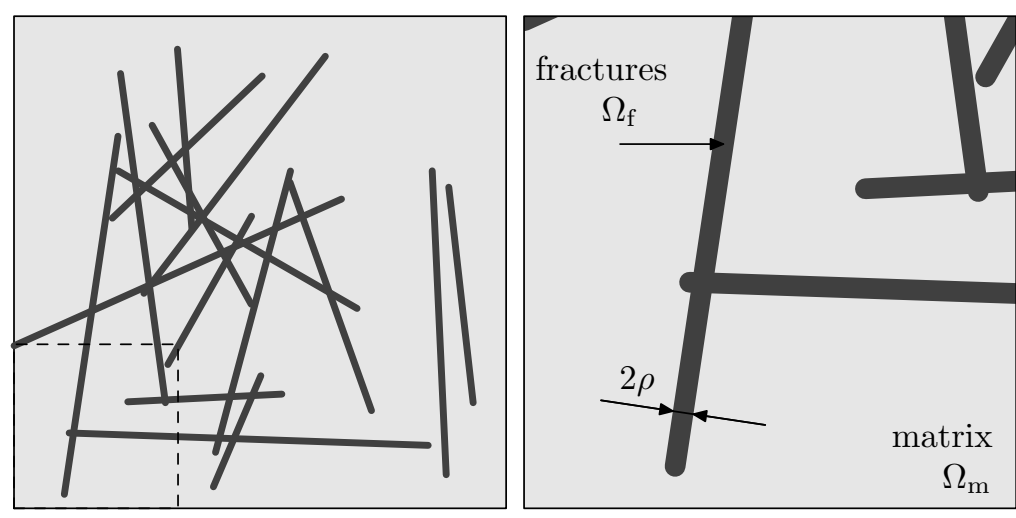

Figure 1: An example of a network of fissures (with zoom)

The simplest equation giving the pressure $p(t, x)$ of a fluid in such a medium at time $t$ and in the point $x$ is:

$$
\frac{\partial p(t, x)}{\partial t}=A p(t, x)
$$

where

$$
A=\sum_{i, j=1}^{2} \frac{\partial}{\partial x_{i}}\left(a_{i, j}(x) \frac{\partial}{\partial x_{j}}\right)
$$

where

$$
a(x)= \begin{cases}a_{-} \times, & \text {if } x \in \Omega_{\mathrm{m}}, \\ a_{+} \times, & \text {if } x \in \Omega_{\mathrm{f}},\end{cases}
$$

with $a_{+} \gg a_{-}$. The coefficient $a$ represents the diffusivity of the rock.

But Equation (1) is written at the scale of the pores, whereas an oil tank can have length of several kilometers. One of the methods to study the 
pressure consists in transforming (1) into a system:

$$
\left\{\begin{aligned}
\Phi_{\mathrm{m}} \frac{\partial P_{\mathrm{m}}}{\partial t}=a_{-} \triangle P_{\mathrm{m}}-\alpha\left(P_{\mathrm{m}}-P_{\mathrm{f}}\right), & \Phi_{\mathrm{m}}=\frac{\operatorname{Meas}\left(\Omega_{\mathrm{m}}\right)}{\operatorname{Meas}(\Omega)} \\
\Phi_{\mathrm{f}} \frac{\partial P_{\mathrm{f}}}{\partial t}=a_{+} \triangle P_{\mathrm{f}}+\alpha\left(P_{\mathrm{m}}-P_{\mathrm{f}}\right), & \Phi_{\mathrm{f}}=\frac{\operatorname{Meas}\left(\Omega_{\mathrm{f}}\right)}{\operatorname{Meas}(\Omega)}
\end{aligned}\right.
$$

where $P_{\mathrm{m}}$ and $P_{\mathrm{f}}$ are the mean pressure in the matrix and the fissures over a given volume $V$ :

$$
\begin{aligned}
P_{\mathrm{m}}(t, x) & =\frac{1}{\operatorname{Meas}\left(V \cap \Omega_{\mathrm{m}}\right)} \int_{x+\left(V \cap \Omega_{\mathrm{m}}\right)} p(t, y) \mathrm{d} y \\
\text { and } P_{\mathrm{f}}(t, x) & =\frac{1}{\operatorname{Meas}\left(V \cap \Omega_{\mathrm{f}}\right)} \int_{x+\left(V \cap \Omega_{\mathrm{f}}\right)} p(t, y) \mathrm{d} y .
\end{aligned}
$$

The coefficient $\alpha$ is called the exchange coefficient. And the model (4) is the double porosity model, here presented in permanent regime (steady state approximation) [WR63]. A more complicated version of the double porosity model may be found in [QW96a, $\mathrm{CFL}^{+} 00$ ] (see also references within), with some numerical analysis.

We deal in this report with the case where the ratio $a_{+} / a_{-}$is very large. The oil is initially in the matrix, but, when moving, the oil stay essentially in the fissures net. The term $a_{-} \triangle P_{\mathrm{m}}$ is negligible in front of the other terms. The Laplace transform of the average of the pressure $P_{\mathrm{f}}(t, x)$ is solution to

$$
\triangle \mathscr{L} P_{\mathrm{f}}(s, x)=s f(s) \mathscr{L} P_{\mathrm{f}}(t, x),
$$

where, in permanent regime, $f$ is the function

$$
f(s)=\frac{\Phi_{\mathrm{f}} \Phi_{\mathrm{m}} s+\alpha}{\Phi_{\mathrm{m}} s+\alpha}
$$

In the permanent regime, and when $a_{+}$is considered as infinite, it has been proved in [NEL01] that if $\langle t\rangle$ is the mean of the first hitting time of the fissures for Brownian particles at speed $2 a_{-}$launched uniformly in the matrix, then

$$
\alpha=\frac{\Phi_{\mathrm{m}}}{\langle t\rangle} \simeq \frac{1}{\langle t\rangle} \text { since } \Phi_{\mathrm{m}} \simeq 1 .
$$

The idea here is to replace the algorithm proposed by B. NeEtinger (of the IFP) and his coauthors using random walks in [NE00, NEQ01, NEL01] by an algorithm using exact computation on some distribution of diffusion processes. This algorithm using continuous time random walks is itself an 
adaptation of the method introduced first by J.F. McCarthy [McC90, McC91, McC93a, McC93b].

The fundamental characteristic of such an algorithm is that it is free from grid generation, which is the most expensive step of the approach either by random walks or by analytical approaches, which need discretization (see e.g., $\left.\left[\mathrm{CFL}^{+} 00\right]\right)$.

It is known that the operator $A$ with domain

$$
\operatorname{Dom}(A)=\left\{u \in \mathrm{H}_{0}^{1}\left(\mathbb{R}^{2}\right) \mid A u \in \mathrm{L}^{2}\left(\mathbb{R}^{2}\right)\right\}
$$

is the infinitesimal generator of a Feller semi-goup, and then that a diffusion process admits $A$ as generator. Furthermore this process is conservative and continuous (see [Str88, Lej00] for example). It may also be studied by the theory of Dirichlet forms [FOT94, MR91]. The articles [GOO87, Por79a, Por79b, MT90, MD92] contain some accounts about the semi-group in case of coefficients having discontinuities along hypersurfaces.

The trajectories of the diffusion process are interpreted as the movement of some particle in the media. In the matrix, the particle moves like a Brownian motion with speed $2 a_{-}$. When it is in the fissures, it moves like a Brownian motion with speed $2 a_{+}$. The passage from the matrix to the fissure needs some special treatment, which will not be considered there. However, we may assume that once it has hit the interface between the matrix and the fissure, the particle enters into the last one. A justification of this may be found in [CLR00].

In this first part, we deal with the hitting time of the fissure for some Brownian particles moving in the matrix.

In our algorithm, we iteratively construct some square centered on the particle at a given time, and we draw the time and the exit position from this square, until it reaches the fissure network. Hence, the trajectory of the particle is not simulated. All the difficulty lies in the choice of a "good" square.

From a numerical point of view, this algorithm has the following advantage:

- No grid required.

- The amount of memory required is of order of the amount of memory required to store the description of the fissures.

- Since the particles are simulated independently, the algorithm is easily parallelized. 
F. Campillo and A. Lejay / A Monte Carlo Method witouth grid

- We may use localization technics to work just on some parts of the matrix.

- The code is rather short.

\section{The algorithm}

\subsection{The main work}

Let suppose that the fissures network is of the following form:

$$
\Omega_{\mathrm{f}}=\cup_{i \in F}\left[A_{i}, B_{i}\right],
$$

where $A_{i}$ and $B_{i}$ are point from $\mathbb{R}^{2}$. Here, the fissures are supposed to be of zero width.

The algorithm relies on the simulation of time and position of exit from a domain of simple geometrical shape, namely the square.

The algorithm is the following (a description in pseudo-language of our algorithm may be found in Appendix A):

\section{Algorithm A : Computation of exit time and position from the matrix.}

A.1 At time $t$, the particle is at a point $P$ of the $\Omega_{\mathrm{m}}$.

A.2 For $i \in F$,

A.2.1 one computes the projected position $H_{i}$ of the point $P$ on the line including the segment $\left[A_{i}, B_{i}\right]$.

A.2.2 if the point $H_{i}$ belongs to $\left[A_{i}, B_{i}\right]$, then let $\delta_{i}=\mathrm{d}\left(P, H_{i}\right)$. Otherwise, let:

$$
\delta_{i}=\min \left\{\mathrm{d}\left(P, A_{i}\right), \mathrm{d}\left(P, B_{i}\right)\right\} .
$$

The value $\delta_{i}$ is the minimal distance of $P$ to $\left[A_{i}, B_{i}\right]$.

A.3 Let $i \in F$ such that $\delta_{i}=\min _{j \in F} \delta_{j}$.

A.3.1 If the point $H_{i}$ belongs to the segment $\left[A_{i}, B_{i}\right]$, then one seeks if it possible to build a square $C$ of which one on the sides rests on the segment $\left[A_{i}, B_{i}\right]$. For that, it is enough for the distance $\delta_{i}$ to be smaller than $\min \left\{\mathrm{d}\left(A_{i}, H_{i}\right), \mathrm{d}\left(B_{i}, H_{i}\right)\right\}$. If not, we go to step A.3.2. 
F. Campillo and A. Lejay / A Monte Carlo Method witouth grid

In this case, $C$ is the square of center $P$ and one on the sides rests on $\left[A_{i}, B_{i}\right]$.

Then, it should be checked if the square $C$ does not intersect any other fissure. It is enough to test this for that all those whose distance $\delta_{j}$ is smaller than $\sqrt{2} \delta_{i}$.

If the interior of the square $C$ intersects another fissure, we go to step A.3.2, else we go to step A.4.

A.3.2 Let $C$ be a square of center $P$ and diagonal length $2 \delta_{i}$.

A.4 We simulate the exit position $P^{\prime}$ and the exit time $\delta t$ from $C$ for a Brownian particle with speed $2 a_{-}$.

If $C$ is a square and the side reached is the one contained in $\left[A_{i}, B_{i}\right]$, then the algorithm stops and returns the position $P^{\prime}$ and the time $t+\delta t$. If not, we return to step A.1 with the new position $P^{\prime}$ and the new time $t+\delta t$.

Figure 2 shows some steps of the simulation of our algorithm A (see also Algorithm 2 in Appendix A). 
F. Campillo and A. Lejay / A Monte Carlo Method witouth grid
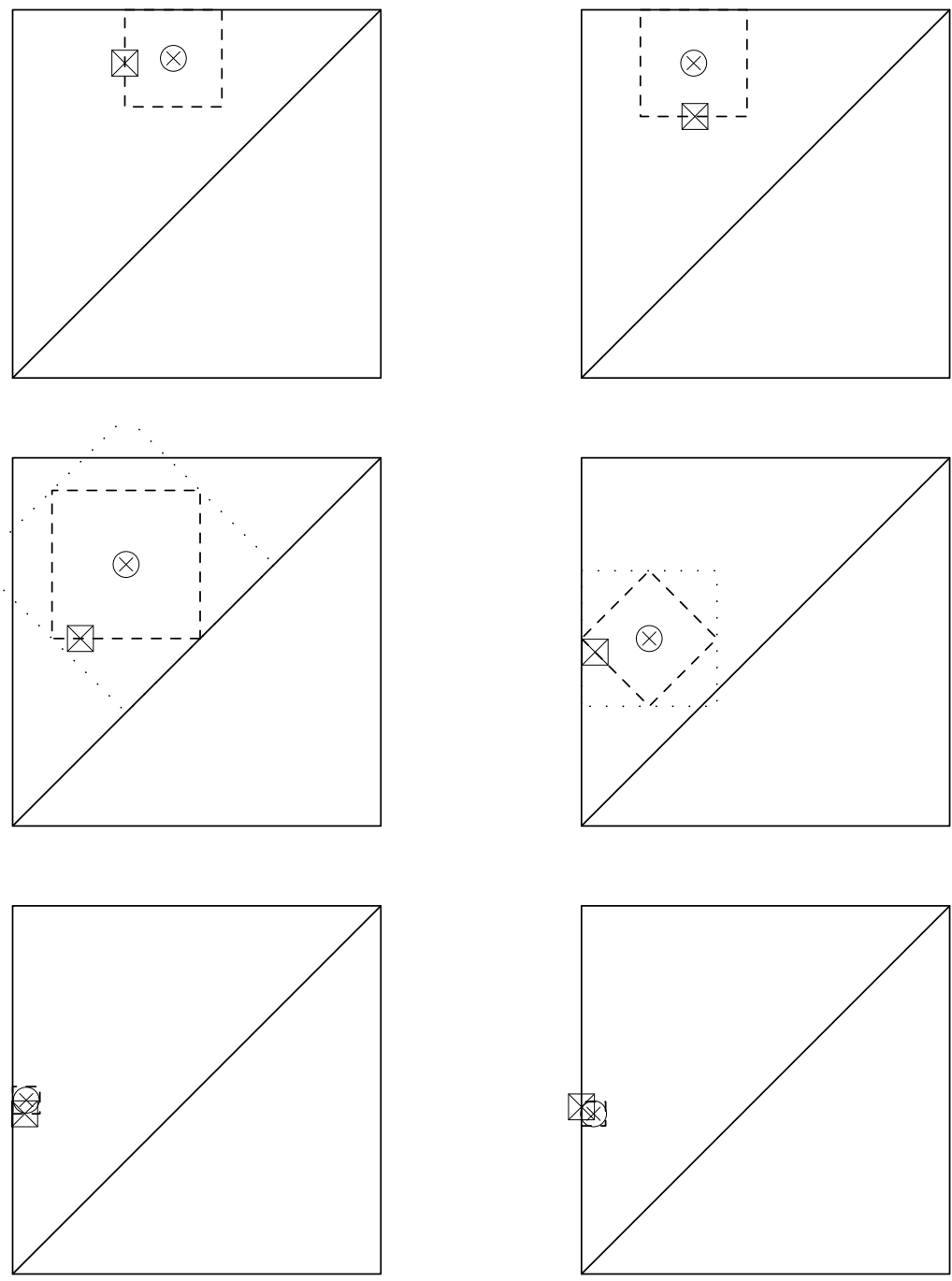

Figure 2: Illustration of the hitting-time simulation algorithm: The circledcross gives the position of the particle at the beginning of the step. The squared-cross gives the position of the particles at the end of the step. The dashed square is the square given whose exit time and position is simulated, while the dotted square is the square first constructed, but intersecting some fissures and then rejected. 


\subsection{Exiting from a square}

We remark that in the previous algorithm, we need to simulate random variables giving us the first time $\tau^{(2)}$ at which a stochastic process $\sqrt{2 a_{-}} W$ exits from a square when it starts at its center, together with the position $\sqrt{2 a_{-}} W_{\tau^{(2)}}$, where $W=\left(W^{(1)}, W^{(2)}\right)$ is a standard $2 d$-Brownian Motion.

In fact, $c W_{t / c^{2}}$ is again a Standard Brownian Motion for any $c>0$, and the distribution of $W$ is invariant under rotation. We may then assume that $2 a_{-}=1$ and that the square is $[-1,1]^{2}$. Hence, we are interested by the joint distribution of

$$
\tau^{(2)}=\inf \left\{t \geqslant 0|| W_{t}^{(i)} \mid=1 \text { for } i=1 \text { or } i=2\right\} \text { and } W_{\tau^{(2)}} .
$$

\subsubsection{Simulation of the exit time from the square}

We have first to simulate the exit time from the square

$$
C=\left\{\left(x^{(1)}, x^{(2)}\right) \in \mathbb{R}^{d}|| x^{(i)} \mid \leqslant 1 \text { for } i=1,2\right\} .
$$

If $\mathscr{P}_{2}$ is the distribution function of $\tau^{(2)}$, then [MT99, Lemma 4.1, p. 741]

$$
\mathscr{P}_{2}(t)=\mathbb{P}\left[\tau^{(2)}<t\right]=1-(1-\mathscr{P}(t))^{2}
$$

where

$$
\mathscr{P}(t)=\mathbb{P}\left[\tau^{(1)}<t\right] \text { with } \tau^{(1)}=\inf \left\{t \geqslant 0 \|\left|W_{t}^{(1)}\right|=1\right\} .
$$

Hence, if $U$ is a uniform random variable on $[0,1], \mathscr{P}^{-1}(1-\sqrt{U})$ is distributed as $\tau^{(2)}$.

The following formulas are borrowed from [MT99, Lemma 3.1, p. 737] and will be used to compute the distribution function $\mathscr{P}$ numerically:

$$
\begin{aligned}
& \mathscr{P}(t)=1-\frac{4}{\pi} \sum_{k=0}^{+\infty} \frac{(-1)^{k}}{2 k+1} \exp \left(-\frac{1}{8} \pi^{2}(2 k+1)^{2} t\right), t>0, \\
& \mathscr{P}(t)=2 \sum_{k=0}^{+\infty}(-1)^{k} \operatorname{erfc} \frac{2 k+1}{\sqrt{2 t}}, t>0
\end{aligned}
$$

where erfc is the complementary of the error function:

$$
\operatorname{erfc}(x)=\frac{2}{\sqrt{\pi}} \int_{x}^{+\infty} \exp \left(-y^{2}\right) \mathrm{d} y .
$$

This distribution function $\mathscr{P}$ has density equal to

$$
\begin{aligned}
& \mathscr{P}^{\prime}(t)=\frac{\pi}{2} \sum_{k=0}^{+\infty}(-1)^{k}(2 k+1) \exp \left(-\frac{1}{8} \pi^{2}(2 k+1)^{2} t\right), t>0, \\
& \mathscr{P}^{\prime}(t)=\frac{2}{\sqrt{2 \pi t^{3}}} \sum_{k=0}^{+\infty}(-1)^{k}(2 k+1) \exp \left(-\frac{1}{2 t}(2 k+1)^{2}\right), t>0 .
\end{aligned}
$$


Formulae (6a) and (7a) are suitable for calculations under large $t$, when $(6 \mathrm{~b})$ and $(7 \mathrm{~b})$ are suitable under small $t$.

\subsubsection{Exit position from the square}

If one of the component of the 2-dimensional Brownian Motion is the first to hit a boundary of $C$ at time $\tau$, then the other remains in the interval $[-1,1]$ during $\left[0, \tau^{(2)}\right]$. Hence, to know $W_{\tau^{(2)}}$, we compute the conditional probability

$$
\mathscr{Q}(\beta, t)=\mathbb{P}\left[W_{t}<\beta|| W_{s} \mid<1,0<s<t\right] .
$$

Using the computations in [MT99],

$$
\begin{aligned}
\mathscr{Q}(\beta, t)= & \frac{1}{1-\mathscr{P}(t)} \frac{2}{\pi} \sum_{k=0}^{+\infty} \frac{1}{2 k+1}\left((-1)^{k}+\sin \frac{\pi(2 k+1) \beta}{2}\right) \\
& \times \exp \left(-\frac{1}{8} \pi^{2}(2 k+1)^{2} t\right), \\
\mathscr{Q}(\beta, t)= & \frac{1}{1-\mathscr{P}(t)} \\
& \times \sum_{k=0}^{+\infty} \frac{(-1)^{k}}{2}\left(\operatorname{erfc} \frac{2 k-1}{\sqrt{2 t}}-\operatorname{erfc} \frac{2 k+\beta}{\sqrt{2 t}}\right. \\
& \left.\quad-\operatorname{erfc} \frac{2 k+2-\beta}{\sqrt{2 t}}+\operatorname{erfc} \frac{2 k+3}{\sqrt{2 t}}\right) .
\end{aligned}
$$

As for $\mathscr{P},(8 \mathrm{a})$ is better for large $t$, when $(8 \mathrm{~b})$ is suitable for small $t$. Of course, $\mathscr{Q}$ admits a smooth density, which is also expressible as series.

\subsection{Simulation of exit time and position}

We have now all the element to provide the algorithm to simulate $\left(\tau^{(2)}, W_{\tau^{(2)}}\right)$, when $C$ is the square $[-1,1]^{2}$ [MT99, Theorem 4.1, p. 743].

We assume that ideally, the random variables generated by the function uniform are independent.

If $C$ is the square with edges $A_{1}, A_{2}, A_{3}$ and $A_{4}$ whose edge length is $2 d$, then the time and position is given by the following algorithm: 
F. Campillo and A. Lejay / A Monte Carlo Method witouth grid

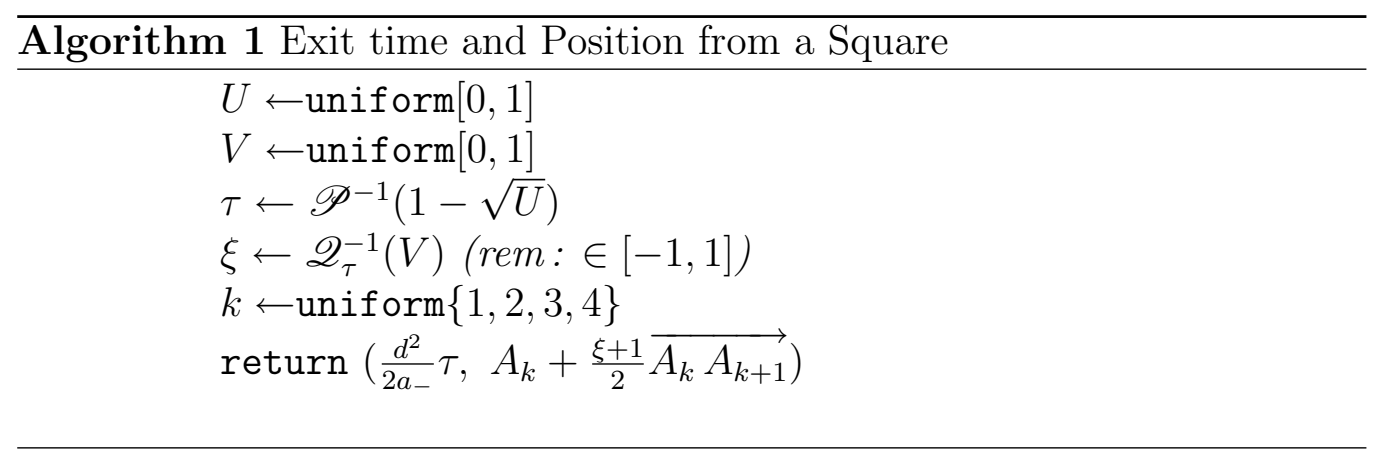

Figures 3 and 4 represent the distribution function $\mathscr{P}$ and its density $\mathscr{P}$, while Figures 5 and 6 show the curve of the distribution function of $\mathscr{Q}(\cdot, t)$ with its density $\mathscr{Q}^{\prime}(\cdot, t)$ for some values of $t$.

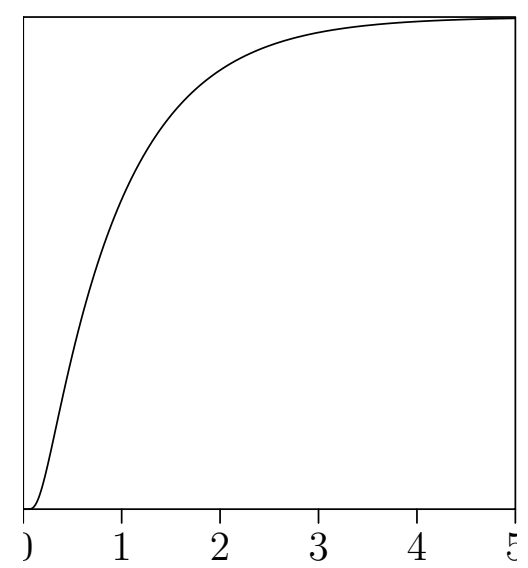

Figure 3: Distribution function $\mathscr{P}$

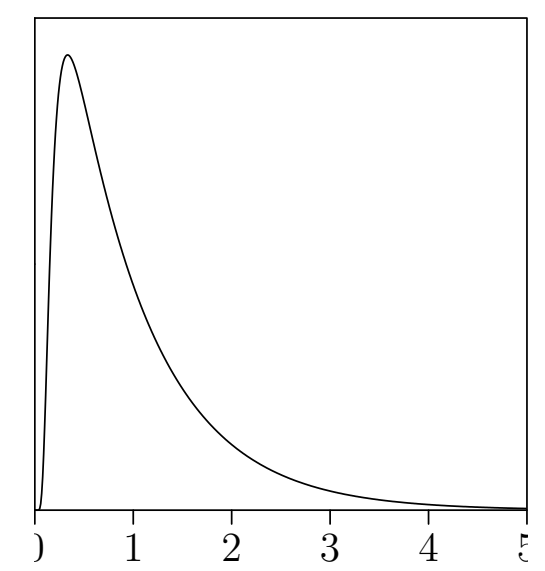

Figure 4: Density $\mathscr{P}^{\prime}$ of the distribution function $\mathscr{P}$ 
F. Campillo and A. Lejay / A Monte Carlo Method witouth grid

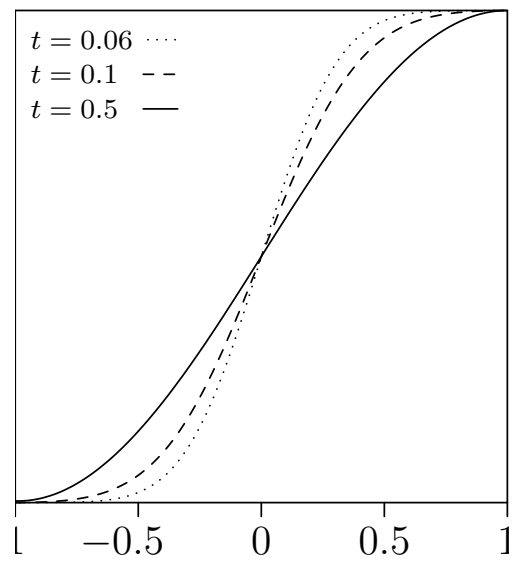

Figure 5: Distribution function $\mathscr{Q}$

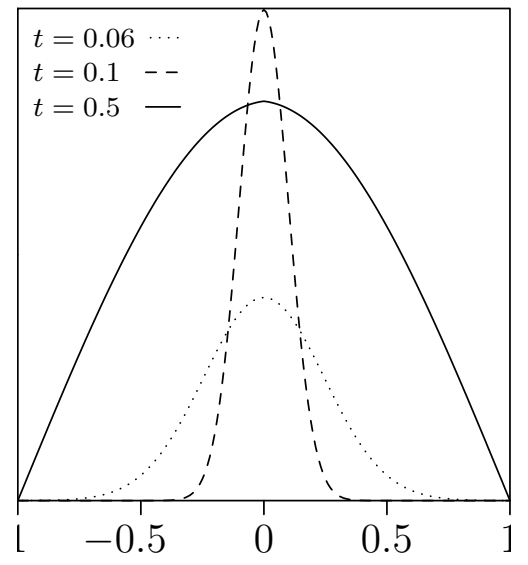

Figure 6: Density $\mathscr{Q}^{\prime}$ of the distribution function $\mathscr{Q}$

\section{$3 \quad$ Numerical results}

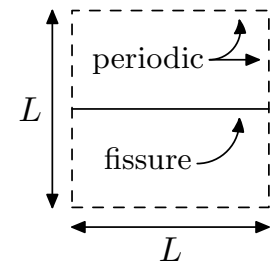

Figure 7: Layered media

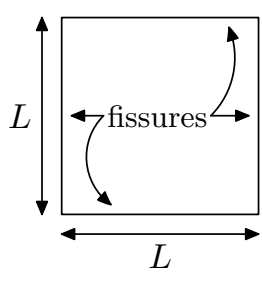

Figure 8: Sugar box

\subsection{The layered media}

The media is infinite in each direction, and is crossed by some horizontal fractures. The distance between a fracture and a fissure is equal to $L$ (See Figure 7). The exchange coefficient is known in this simple case [QW96b], and is equal to

$$
\alpha=\frac{12 a_{-}}{L^{2}} .
$$

In the case of $L$ is equal to 1 and $a_{-}=1$, the theoretical value $\langle t\rangle=$ 0.0833. The experiments in Table 1 shows that the methods provides some quite good results. 
F. Campillo and A. Lejay / A Monte Carlo Method witouth grid

\begin{tabular}{|c|ccccc|c|c|}
\hline Simulations & $10^{2} \cdot \bar{t}_{1}$ & $10^{2} \cdot \bar{t}_{2}$ & $10^{2} \cdot \bar{t}_{3}$ & $10^{2} \cdot \bar{t}_{4}$ & $10^{2} \cdot \bar{t}_{5}$ & $10^{2} \cdot m_{5}$ & $10^{3} \cdot \sigma_{5}$ \\
\hline$n=1,000$ & 8.33 & 8.58 & 7.88 & 8.11 & 8.17 & 8.21 & 2.60 \\
$n=2,000$ & 8.53 & 8.11 & 8.16 & 8.75 & 8.13 & 8.33 & 2.92 \\
$n=5,000$ & 8.44 & 8.33 & 8.31 & 8.34 & 8.16 & 8.31 & 1.04 \\
$n=10,000$ & 8.15 & 8.36 & 8.42 & 8.38 & 8.23 & 8.31 & 1.13 \\
\hline
\end{tabular}

Table 1: Mean of $n$ experiments for the layered media with $L=1$ and $a_{-}=1$. We set $m_{5}=\left(\bar{t}_{1}+\cdots+\bar{t}_{5}\right) / 5$ and $\sigma_{5}=\operatorname{sd}\left(\bar{t}_{1}, \ldots, \bar{t}_{5}\right)$.

In Table 2, the dependence of $L$ is studied. In fact, our algorithm is scale-independent, and the results behave as expected when $L$ increases.

\begin{tabular}{|c|r|r|r|}
\hline$L$ & \multicolumn{1}{|c|}{$\alpha$} & \multicolumn{1}{|c|}{$1 /\langle t\rangle$} & $10^{2} \times\langle t\rangle$ \\
\hline 1 & 12.00 & 12.14 & 8.234 \\
2 & 3.00 & 2.89 & 34.570 \\
3 & 1.33 & 1.33 & 75.125 \\
4 & 0.75 & 0.74 & 135.389 \\
5 & 0.48 & 0.50 & 198.496 \\
\hline
\end{tabular}

Table 2: Five experiments for 1,000 simulations in the layered media in function of $L$

\subsection{The sugar box}

In this case, the media is composed of porous square box surrounded by some fractures. We have just to study the mean of the exit time for some particles launched with uniform distribution on a square of size $L \times L$. The value of $\alpha$ computed by Warren and Root [WR63] is $\alpha=28.44 / L^{2}$. When $L=1$, the theoretical value of the exit time is

$$
\langle t\rangle=0.0351
$$

Experiments in Table 3 show that this algorithm also provides some good results. Furthermore, the average number of steps is close to 4 , as we might expect. 
F. Campillo and A. Lejay / A Monte Carlo Method witouth grid

\begin{tabular}{|c|ccccc|c|c|}
\hline Simulations & $10^{2} \cdot \bar{t}_{1}$ & $10^{2} \cdot \bar{t}_{2}$ & $10^{2} \cdot \bar{t}_{3}$ & $10^{2} \cdot \bar{t}_{4}$ & $10^{2} \cdot \bar{t}_{5}$ & $10^{2} \cdot m_{5}$ & $10^{3} \cdot \sigma_{5}$ \\
\hline$n=1,000$ & 3.36 & 3.51 & 3.63 & 3.45 & 3.48 & 3.49 & 1.00 \\
$n=2,000$ & 3.39 & 3.61 & 3.35 & 3.54 & 3.45 & 3.47 & 1.08 \\
$n=5,000$ & 3.53 & 3.59 & 3.52 & 3.53 & 3.51 & 3.54 & 0.29 \\
$n=10,000$ & 3.49 & 3.56 & 3.56 & 3.41 & 3.49 & 3.50 & 0.63 \\
\hline
\end{tabular}

Table 3: Mean of $n$ experiments for the sugar box with $L=1$ and $a_{-}=1$. We set $m_{5}=\left(\bar{t}_{1}+\cdots+\bar{t}_{5}\right) / 5$ and $\sigma_{5}=\operatorname{sd}\left(\bar{t}_{1}, \ldots, \bar{t}_{5}\right)$.

Table 4 shows that the dependence in the size $L$ of the sugar box is kept.

\begin{tabular}{|c|r|r|c|}
\hline$L$ & \multicolumn{1}{|c|}{$\alpha$} & \multicolumn{1}{c|}{$1 /\langle t\rangle$} & $10^{2} \times\langle t\rangle$ \\
\hline 1 & 28.44 & 30.23 & 3.308 \\
2 & 7.11 & 6.74 & 14.827 \\
3 & 3.16 & 3.35 & 29.832 \\
4 & 1.78 & 1.87 & 53.526 \\
5 & 1.14 & 1.13 & 88.816 \\
\hline
\end{tabular}

Table 4: Five experiments for 1,000 simulations in the sugar box in function of $L$

\subsection{Non trivial case}

In this case, the fissures net is more complex: see Figure 9.

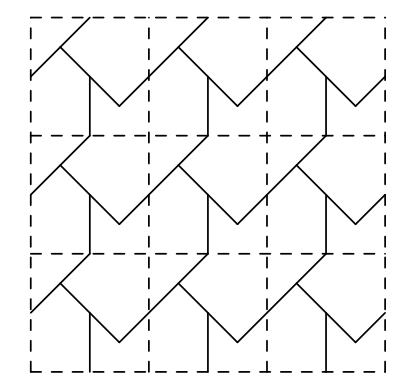

Figure 9: A non-trivial fissures net

In $\left[\mathrm{CFL}^{+} 00\right]$, the exchange coefficient is computed using a finite-volume method, and when $L=1$ and $a_{-}=1$, its value is

$$
\alpha_{\text {f.v.m. }}=35.03 \text {. }
$$


We have to note that the model proposed in $\left[\mathrm{CFL}^{+} 00\right]$ is more complex than our, since the width of the fissure is not assume to be equal to 0 , and the diffusion coefficient $a_{+}$in the fissure is not assumed to be infinite.

Using the random walk method proposed in [NE00, NEQ01, NEL01], the same authors have found a value

$$
\alpha_{\text {r.w. }}=34.18
$$

for this geometry [NE98]. Here again, the width of the fissures is not neglected.

With 20,000 experiments with the conditions $L=1$ and $a_{-}=1$, we found a value of

$$
\bar{t}=0.028 \text { and } \alpha=\frac{1}{\bar{t}}=35.70 .
$$

The average number of steps is 7.4, with a standard deviation of 6.9. Our value of $\alpha$ is close to the value of the exchange coefficients given by the other methods.

\section{A The algorithm in pseudo-language}

The following functions are used in Algorithm 2:

Square $(P, H)$ : returns a square whose center is $P$ and for which the the middle point of some edge is $H$.

Square_diag $(P, H)$ : returns a square whose center is $P$ and for which the point $H$ is one of its corner.

ExitFromSquare $(C)$ : returns the exit time at which the Brownian particle at a given speed starting from the center of $C$ goes out from the square $C$. It also returns the corresponding position.

\section{References}

$\left[\mathrm{CFL}^{+} 00\right]$ Y. Caillabet, P. Fabrie, P. Landereau, B. Netinger and M. QuintaRD. Implementation of a finite-volume method for the determination of effective parameters in fissured porous media. Numer. Methods Partial Differential Equations, vol. 16, nº2, pp. 237-263, 2000. 


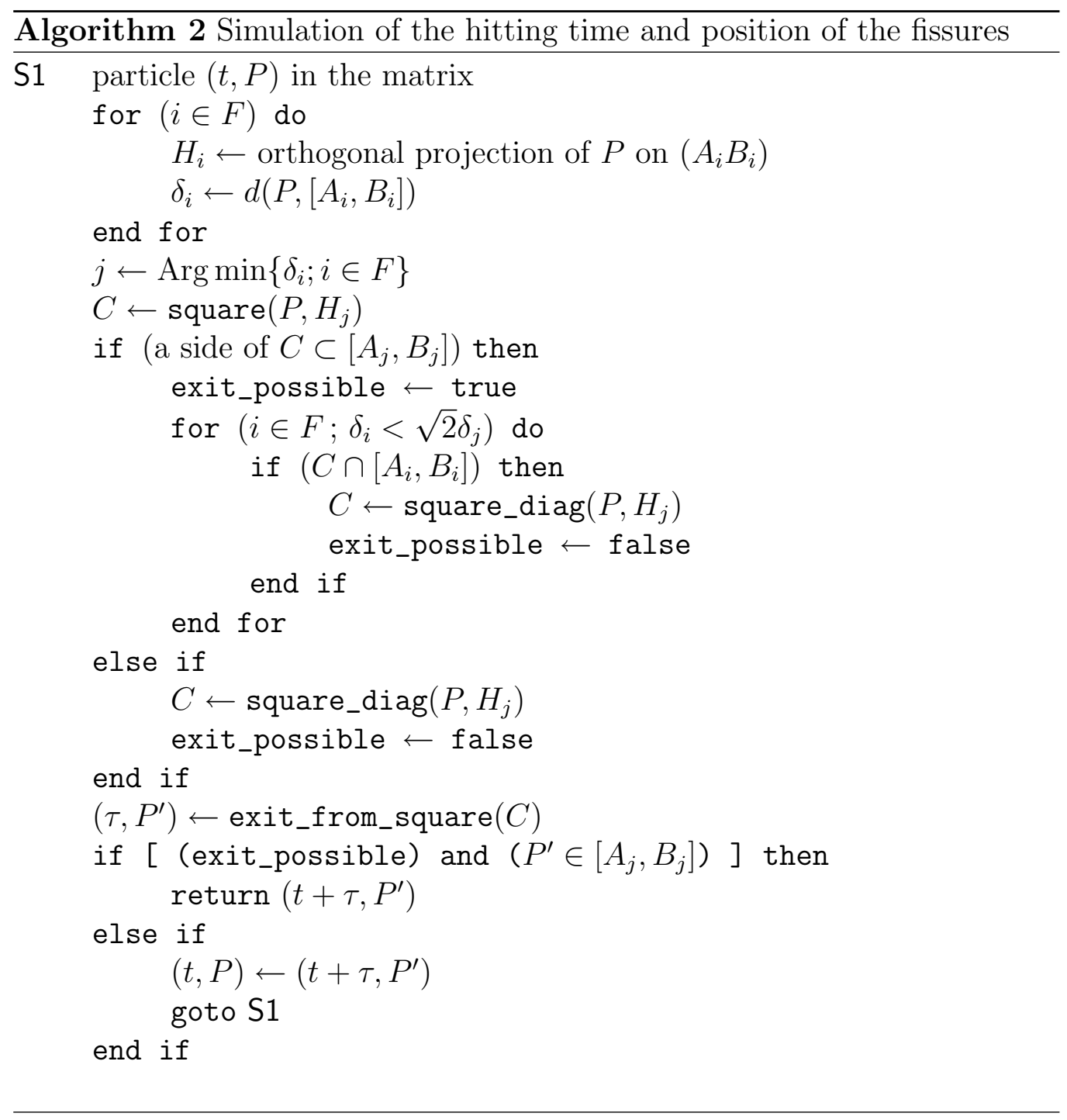

[ClR00] F. Campillo, A. Lejay and E. Remy. A Monte-Carlo Method without Grid to Compute the Exchange Coefficient in the Double Porosity Model. Part II: In the Fissures. Preliminary Version, 2000 .

[Fot94] M. Fukushima, Y. Oshima and M. Takeda. Dirichlet Forms and Symmetric Markov Process. De Gruyter, 1994.

[GO087] B. Gaveau, M. Okada and T. Okada. Second order differential operators and Dirichlet integrals with singular coefficients. I: Functional calculus of one-dimensional operators. Tôhoku Math. J.(2), vol. 39, nº4, pp. 465-504, 1987. 
F. Campillo and A. Lejay / A Monte Carlo Method witouth grid

[Lej00] A. LEJAY. Méthodes probabilistes pour l'homogénéisation des opérateurs sous forme-divergence : cas linéaires et semi-linéaires. PhD thesis, Université de Provence, 2000. [URL: www.iecn. u-nancy.fr/ lejay/].

[McC90] J.F. MCCARTHY. Effective permeability of sandstone-shale reservoirs by random walk method. J. Phys. A: Math. Gen., vol. 23, pp. L445-L451, 1990.

[McC91] J.F. MCCARThy. Analytical models of the effective permeability of sand-shale reservoirs. Geophys. J. Int., vol. 105, pp. 513-527, 1991.

[McC93a] J.F. MCCARTHY. Continuous-time random walks on random media. J. Phys. A: Math. Gen., vol. 26, pp. 2495-2503, 1993.

[McC93b] J.F. MCCARTHY. Reservoir characterization : Efficient randomwalk methods for upscaling and image selection. In Proceedings of the SPE Asia Pacific Oil $\& 5$ Gas Conference $\mathscr{E}$ Exhibition. Singapore, 8-10 February, pp. 159-171, 1993.

[MD92] M. Mastrangelo and D. Dehen. Opérateurs différentiels paraboliques à coefficients continus par morceaux et admettant un drift généralisé. Bull. Sci. Math., vol. 116, pp. 67-93, 1992. $2^{\text {ème }}$ série.

[MR91] Z. MA and M. RöcKNER. Introduction to the Theory of (NonSymmetric) Dirichlet Forms. Universitext. Springer-Verlag, 1991.

[MT90] M. Mastrangelo and M. TAlbi. Mouvement brownien asymétriques modifiés en dimension finie et opérateurs différentiels à coefficients discontinus. Probability and Mathematical Statistics, vol. 11, pp. 47-78, 1990. Fasc. 1.

[MT99] G.N. Milstein and M.V. Tretyakov. Simulation of a spacetime bounded diffusion. Ann. Appl. Probab., vol. 9, no3, pp. 732$779,1999$.

[NE98] B. Netinger and T. Estebenet. Application of random walk methods on unstructured grids to up-scale fractured reservoirs. ECMOR VI Conf, Scotland, 1998.

[NE00] B. Netinger and T. EstéBenet. Up-Scaling of Double Porosity Fractured Media Using Continuous-Time Random Walks Methods. Transport in Porous Media, vol. 39, nº3, pp. 315-337, 2000. 
F. Campillo and A. Lejay / A Monte Carlo Method witouth grid

[NeL01] B. Netinger, T. Estebenet and P. Landereau. A direct determination of the transient exchange term of fractured media using a continuous time random walk method. Transport in Porous Media, vol. 44, nº3, pp. 539-557, 2001.

[NEQ01] B. Netinger, T. Estebenet and M. Quintard. Up-scaling flow in fractured media: equivalence between the large scale averaging theory and the continuous time random walk method. Transport in Porous Media, vol. 43, n³, pp. 581-596, 2001.

[Por79a] N.I. Portenko. Diffusion processes with a generalized drift coefficient. Theory Probab. Appl., vol. 24, nº1, pp. 62-78, 1979.

[Por79b] N.I. Portenko. Stochastic Differential Equations with generalized drift vector. Theory Probab. Appl., vol. 24, nº2, pp. 338-353, 1979.

[QW96a] M. Quintard and S. Whitaker. Transport in chemically and mechanically heterogeous porous media. I: Theoritical development of region-averaged equations for sligthly compressible singlephase flow. Adv. Water Ressources, vol. 19, nº1, pp. 29-47, 1996.

[QW96b] M. Quintard and S. Whitaker. Transport in chemically and mechanically heterogeous porous media. II: Comparisons with numerical experiments for slightly compressible single-phase flow. Adv. Water Ressources, vol. 19, pp. 49-60, 1996.

[Str88] D.W. Stroock. Diffusion Semigroups Corresponding to Uniformly Elliptic Divergence Form Operator. In Séminaire de Probabilités XXII, vol. 1321 of Lecture Notes in Math., pp. 316-347. Springer-Verlag, 1988.

[WR63] J.E. WARren and P.J. Root. The Behavior of Naturally Fractured Reservoirs. Soc. Petrol. Eng. J., vol. 3, n³, pp. 245-255, 1963. 